\title{
ON A THEOREM OF RÅDSTRÖM
}

\section{P. ERDÖS}

The purpose of this note is to give a new and simplified proof of the following theorem.

Theorem. Let $f(z)=\sum_{\nu=0}^{\infty} a_{v} z^{\nu}$ be an entire function. Denote $M(r)$ $=\max _{|z| \leqq r}|f(z)|$. Assume that $\lim$ sup $\log M(r) / r=\infty$. Then there exists $\omega_{\nu}, \nu=0,1, \cdots$, with $\left|\omega_{\nu}\right|=1$, so that the origin is a limit point of the roots of the derivatives of $k(z)=\sum_{\nu=0}^{\infty} \omega_{\nu} a_{\nu} z^{\nu}$.

In other words the theorem holds if the order $\rho$ of $f(z)$ is greater than 1 or if $\rho=1$ and $f(z)$ is of maximal type.

This theorem is due to Rådström and was proved by him for the case $\rho>1$ in a recent note. ${ }^{1}$ The result as announced here is best possible with respect both to order and to type, as is shown by the example $e^{c z}$, where $c$ is a constant (cf. footnote 1, p. 400).

We need the following two lemmas.

LEMMA 1. Let $\sum_{\nu=0}^{\infty} a_{\nu} z^{\nu}$ be a power series with radius of convergence $R \leqq \infty$ and such that $\left|a_{0} / a_{1}\right|<R$. Then it is possible to find $\omega_{0}, \omega_{1}, \cdots$ with $\left|\omega_{\nu}\right|=1$ so that $\sum_{\nu=0}^{\infty} \omega_{\nu} a_{\nu} z^{\nu}$ has a zero $z_{0}$ with $\left|z_{0}\right| \leqq\left|a_{0} / a_{1}\right|$.

Proof. We put $\omega_{0}=\omega_{1}=1$ and $a_{0}+a_{1} z=P_{1}(z)$. Obviously $P_{1}(z)$ has a zero with the required property. We proceed by induction. Suppose that we have succeeded in determining $\omega_{0}, \omega_{1}, \cdots, \omega_{n-1}$ such that the polynomial $P_{n-1}(z)=\sum_{\nu=0}^{n-1} \quad \omega_{\nu} a_{\nu} z^{\nu}$ has a zero $z_{0}$ with $\left|z_{0}\right|$ $\leqq\left|a_{0} / a_{1}\right|$. Consider $P_{n-1}(z)+\omega a_{n} z^{n},|\omega|=1$. Three cases may occur:

1. The equation $\left|P_{n-1}(z)\right|=\left|a_{n} z^{n}\right|$ has a solution on $|z|=\left|a_{0} / a_{1}\right|$.

2. $\left|P_{n-1}(z)\right|>\left|a_{n} z^{n}\right|$ for all $z$ with $|z|=\left|a_{0} / a_{1}\right|$.

3. $\left|P_{n-1}(z)\right|<\left|a_{n} z^{n}\right|$ for all $z$ with $|z|=\left|a_{0} / a_{1}\right|$.

In case 1 , it will obviously be possible to choose $\omega$ so that $P_{n-1}(z)$ $+\omega a_{n} z^{n}=0$ on the circle $|z|=\left|a_{0} / a_{1}\right|$. In case $2, P_{n-1}(z)+\omega a_{n} z^{n}$ has by Rouché's theorem as many zeros inside the circle $|z|=\left|a_{0} / a_{1}\right|$ as $P_{n-1}(z)$, that is, at least one, by the induction hypothesis. In case 3 , again by Rouché's theorem, $P_{n-1}(z)+\omega a_{n} z^{n}$ has as many zeros in $|z|=\left|a_{0} / a_{1}\right|$ as $a_{n} z^{n}$, that is, $n$ zeros. In all these cases we can therefore choose $\omega=\omega_{n},\left|\omega_{n}\right|=1$ so that $P_{n-1}(z)+a_{n} \omega_{n} z^{n}$ has a zero in the circle $\left|z_{0}\right|=\left|a_{0} / a_{1}\right|$. Consider now the power series $\sum_{\nu=0}^{\infty} \omega_{\nu} a_{\nu} z^{\nu}$. We know that all its partial sums have zeros in or on the circle $|z|=\left|a_{0} / a_{1}\right|$. As this circle is strictly inside the circle of convergence

Received by the editors January 30, 1950.

${ }^{1}$ H. Rådström, Proc. Nat. Acad. Sci. U.S.A. vol. 35 (1949) pp. 399-404. 
the same must hold for the infinite series, which proves the lemma.

LеммA 2. Let $\sum_{\nu=0}^{\infty} a_{\nu} z^{\nu}$ satisfy the conditions of Lemma 1, and let $\epsilon$ be a positive number. Then there exists an integer $n$ and numbers $\omega_{0}, \omega_{1}, \cdots, \omega_{n}$ with $\left|\omega_{\nu}\right|=1$ such that the series $\sum_{\nu=0}^{\infty} \omega_{\nu} a_{\nu} z^{\nu}$ has $a$ zero in the circle $|z| \leqq\left|a_{0} / a_{1}\right|+\epsilon$, irrespective of the choice of the numbers $\omega_{\text {, for }} \nu \geqq n+1$.

Proof. Let $r$ be a number with $\left|a_{0} / a_{1}\right|<r<\min \left(R,\left|a_{0} / a_{1}\right|+\epsilon\right)$ and such that the series $f(z)=\sum_{\nu=0}^{\infty} \omega_{\nu} a_{\nu} z^{\nu}$ constructed in Lemma 1 has a positive minimum $m$ on the circle $|z|=r$. Put $\delta_{\mu}=\sum_{\nu=\mu+1}^{\infty}\left|a_{\nu}\right| r^{\nu}$. We have $\delta_{\mu} \rightarrow 0$ monotonically. Choose $n$ so large that $2 \cdot \delta_{n}<m$, and let $g(z)$ be any series which coincides with $f(z)$ in the first $n+1$ terms whereas in the rest of the terms arbitrary changes of the arguments are allowed. Obviously $|g(z)-f(z)|<2 \cdot \delta_{n}$ for $|z| \leqq r$. Therefore, by Rouché's theorem, $g(z)$ has as many roots in $|z| \leqq r$ as $f(z)$, that is, at least one (since $\left.r>\left|a_{0} / a_{1}\right|\right)$. This proves the lemma.

In order to prove the theorem we first observe that if lim sup $\log M(r) / r=\infty$, it follows that $\lim$ inf $\left|a_{n} /(n+1) a_{n+1}\right|=0$, for otherwise there would exist a $k>0$ such that for all sufficiently large $n, a_{n+1}<k a_{n} /(n+1)$. Iterating this we would get, for sufficiently large $n, a_{n}<c k^{n} / n$ !, which as is well known implies $\lim \sup \log M(r) / r$ $\leqq k$, an evident contradiction. Therefore there exists a sequence $n_{\nu}$ of integers such that $a_{n_{v}} /(n+1)\left(a_{n_{v}+1}\right) \rightarrow 0$. We also observe that $f^{(n)}(z) / n !=a_{n}+(n+1) a_{n+1} z+\ldots$. Now choose a sequence $\epsilon_{p}$ of positive numbers with $\epsilon_{\nu} \rightarrow 0$. According to Lemma 2 we can find numbers $\omega_{n_{1}}, \omega_{n_{1}+1}, \cdots, \omega_{n_{1}+p_{1}}$ so that if in $f^{\left(n_{1}\right)}(z) / n_{1}$ ! we multiply each coefficient with the corresponding $\omega$, we shall get a function which has a zero in $|z|<\left|a_{n_{1}} /\left(n_{1}+1\right) a_{n_{1}+1}\right|+\epsilon_{1}$, and we shall still be able to choose $\omega_{\mu}$ arbitrarily if $\mu>n_{1}+p_{1}$ without destroying this property. Therefore we can repeat this process, now starting with the smallest $n_{\nu}>n_{1}+p_{1}$. Call that number $m_{2}$ and put $m_{1}=n_{1}$. Then we get a new set of $\omega$ 's, $\omega_{m_{1}}, \cdots, \omega_{m_{2}+p_{2}}$, and if $\mu>m_{2}+p_{2}$ we still have the free choice of the $\omega_{\mu}$. Iterating this process we shall obtain a sequence of nonoverlapping blocks of $\omega$ 's and we complete it if necessary by choosing $\omega_{\nu}$ arbitrarily for those $\nu$ which do not correspond to an $\omega$ in a block. In this way we get a sequence $\omega_{0}, \omega_{1}, \cdots$ and we construct the corresponding power series $k(z)=\sum_{\nu=0}^{\infty} \omega_{\nu} a_{\nu} z^{\nu}$. From the construction and Lemma 2 it is then obvious that $k(z)$ will have the property: $k^{\left(m_{\nu}\right)}(z)$ has a zero $z_{\nu}$ satisfying $z_{\nu}<\left|a_{m_{\nu}} /\left(m_{\nu}+1\right) a_{m_{\nu}+1}\right|$ $+\epsilon_{\nu}$. As the sequence $m_{\nu}$ is a subsequence of $n_{\nu}$ and $\epsilon_{\nu} \rightarrow 0$, it is clear that $z_{\nu} \rightarrow 0$, which proves the theorem.

Princeton, N. J. 